\title{
MODELING THE ANHARMONIC INFRARED EMISSION SPECTRA OF PAHS: APPLICATION TO THE PYRENE CATION
}

\author{
M. Basire ${ }^{1}$, P. Parneix ${ }^{1}$, T. Pino ${ }^{1}$, Ph. Bréchignac ${ }^{1}$ and F. Calvo ${ }^{2}$
}

\begin{abstract}
The IR emission cascade from the pyrene cation due to a broad band optical excitation is simulated using kinetic Monte Carlo. Anharmonicities of the ground electronic state potential energy surface are taken into account in the transition energies, the microcanonical densities of states, and the rate of hydrogen loss through various statistical theories. The emission spectral features of the "3.3", "6.2" and "11.2" $\mu \mathrm{m}$ bands are computed for different blackbody temperatures.
\end{abstract}

\section{Introduction}

Unidentified Infrared Bands (UIBs) correspond to intense IR emission bands in the 3-20 $\mu \mathrm{m}$ spectral range. According to the so-called PAH model (Puget \& Léger 1989; Allamandola et al. 1989); these IR bands, first detected in 1973 (Gillett et al. 1973), are attributed to PAH emission bands, although no conclusive identification of molecular carriers has been achieved yet. In the last years, a large number of astrophysical objects has been observed in this IR spectral range and high resolution spectra are now available (Hony et al. 2001; Peeters et al. 2002; Van Diedenhoven et al. 2004; Draine \& Li 2007). In the photophysics PAH model, the molecule is initially excited by absorption of a visible/UV photon, after which the intramolecular dynamics is driven by non adiabatic couplings. Subsequently, the PAH molecule is vibrationally heated in the ground electronic state and cools down by emission of several IR photons.

Up to now, only very few experimental data have been collected on the IR emission of neutral (Cherchneff \& Barker 1989; Brenner \& Barker 1992; Williams \& Leone 1995; Cook et al. 1996; Cook \& Saykally 1998) and cationic (Kim et al. 2001;

\footnotetext{
${ }^{1}$ Institut des Sciences Moléculaires d'Orsay, CNRS UMR 8214, Université Paris-Sud 11, Bât. 210, 91405 Orsay Cedex, France

${ }^{2}$ LASIM, Université de Lyon and CNRS UMR 5579, 43 Bd. du 11 Novembre 1918, 69622 Villeurbanne Cedex, France
} 
Kim \& Saykally 2002) PAHs after an initial excitation provided by a UV laser or an electron gun. These experiments have found evidence that IR emission spectra are shifted with respect to absorption spectra, the vibrational shift being dependent on the amount of internal energy deposited in the molecule.

From the theoretical point of view, IR emission spectra have been mainly computed in the harmonic limit. In these simulations, the IR radiative cooling of the molecule is monitored using thermal approximations (Léger et al. 1989; Schutte et al. 1993) or a stochastic model (Gillespie 1978; Barker 1983; Mulas 1998; Joblin et al. 2002). More recently, in order to reproduce the shape of the spectral profile, anharmonicities were empirically introduced in the spectral simulation (Cook \& Saykally 1998; Pech et al. 2002), using experimental data on the spectral shifts and broadenings of the IR absoption spectra for neutral PAHs, as a function of increasing temperature (Joblin et al. 1995).

In the present article, we describe a novel theoretical approach to determine the IR radiative cascade. In this method, anharmonicities calculated using firstprinciple methods and second-order perturbation theory are taken into account in all statistical quantities, namely the microcanonical densities of states used in the thermal populations and the dissociation rates, as well as the transition energies themselves.

\section{Model and methods}

We assume that the initial excitation energy, deposited as visible/UV light, is rapidly converted into vibrational energy in the ground electronic state. Our purpose is then to model the (much slower) radiative decay of the molecule through multiple emission in the infrared domain. In absence of collisional relaxation (low density medium), the internal energy remains constant between two successive IR photon emissions, and the physical conditions are those of a microcanonical ensemble. If the time scale between two such emissions is long with respect to intramolecular vibrational redistribution (IVR), then all vibrational states at the available internal energy $E$ are equiprobable.

Because the excitation energy is large with respect to the emitted IR photons, the energy in the electronic ground state is expected to be initially rather high, hence it is important to account for anharmonicities in the description of transition energies, as well as for counting accurate densities of states (DOS's). We have previously developed a computational procedure based on the WangLandau Monte Carlo method (Wang \& Landau 2001) to determine the quantum density of vibrational states of fully coupled polyatomic systems. Briefly, the method starts with a perturbative Dunham expansion of the vibrational energy levels in terms of harmonic wavelengths $\lambda_{k}^{(\mathrm{h})}$ and anharmonic coefficients $\chi_{k \ell}$, as obtained from quantum chemistry calculations. In the present case, all ingredients were determined using density-functional theory, with the hybrid B3LYP potential and the basis set 4-31G, of modest size because of computer limitations for this rather large molecule. The quadratic force field was then sampled by performing a random walk in energy space, the microcanonical DOS being estimated with 

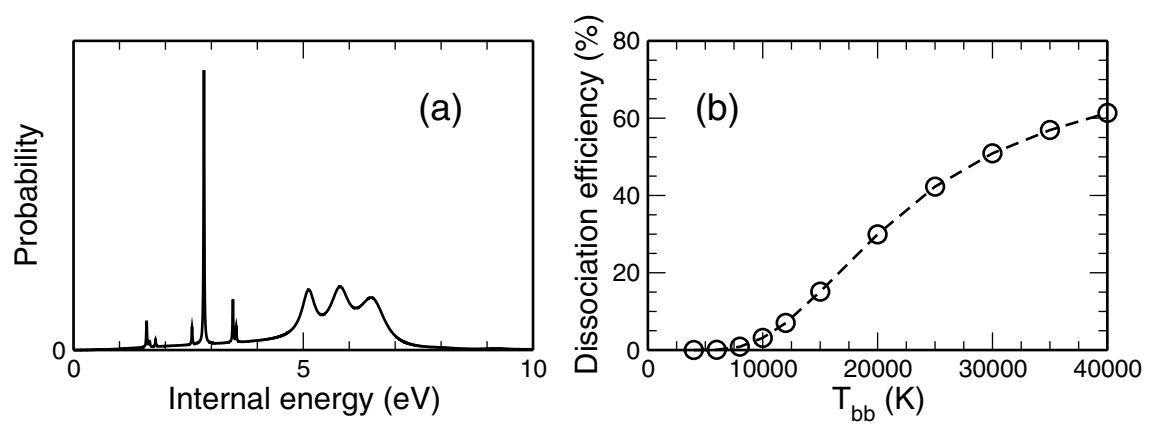

Fig. 1. (a) Distribution of internal excitation energy $P\left(E^{*}\right)$ at blackbody temperature $T_{\mathrm{bb}}=10000 \mathrm{~K}$; (b) dissociation efficiency versus blackbody temperature $T_{\mathrm{bb}}$.

increasing accuracy over a broad energy range (Basire et al. 2008). Knowledge of the DOS also provides a very convenient way of calculating the infrared emission spectrum at microcanonical equilibrium, by accumulating a two-dimensional histogram $\mathcal{I}_{e}(\nu ; E)$ of emission frequency $\nu$ at total energy $E$ along a multicanonical Monte Carlo simulation (Basire et al. 2009,2010). The energy-resolved spectrum is explicited as

$$
\mathcal{I}_{e}(\nu ; E)=\frac{1}{\mathcal{N}(\nu ; E)} \sum_{\text {MC steps }} \sum_{k} n_{k} A_{k}^{(1 \rightarrow 0)} \delta\left(h \nu-\Delta E^{(k)}\right),
$$

where $\mathcal{N}(\nu ; E)$ is the number of entries in the histogram corresponding to the emission of a photon with energy $h \nu$, at total energy $E$. In this equation, $n_{k}$ is the vibrational level of mode $k$ with harmonic frequency $\nu_{k}^{(\mathrm{h})}, \Delta E^{(k)}$ is the transition energy associated with the emission of a single photon of mode $k$, and $n_{k} A_{k}^{(1 \rightarrow 0)}$ is the associated oscillator strength, assuming here an harmonic approximation.

In practice, the microcanonical spectra of the pyrene cation have been computed using the above procedure for vibrational energies ranging from the ground state level $\left(E_{\min }=0\right)$ to $E_{\max }=14 \mathrm{eV}$, with a resolution $\Delta E=100 \mathrm{~cm}^{-1}$ in energy and $\Delta \nu=0.2 \mathrm{~cm}^{-1}$ in emission frequency. Anharmonicities enter the above ingredients through the densities of microcanonical states and thermal populations, and in the transition energies.

Next, a complete IR emission cascade is simulated using a kinetic Monte Carlo (kMC) model (Mulas 1998). At any given energy $E$, several photon emission processes compete with each other and with molecular dissociation. For simplicity, we only include the lowest (most probable) dissociation channel, namely hydrogen loss, for which the dissociation rate $k_{\text {diss }}(E)$ is accurately modeled using phase space theory (Pino et al. 2007). Concerning radiative relaxation, the emission rate $k(\nu ; E)$ for a photon $h \nu$ at internal energy $E$ is simply given by $\mathcal{I}_{e}(\nu ; E)$. Starting at time $t=0$ with an excess energy $E(t=0)=E^{*}$, all possible relaxation events are enumerated, including emission of a single photon from mode $k$ with $n_{k}>0$, 

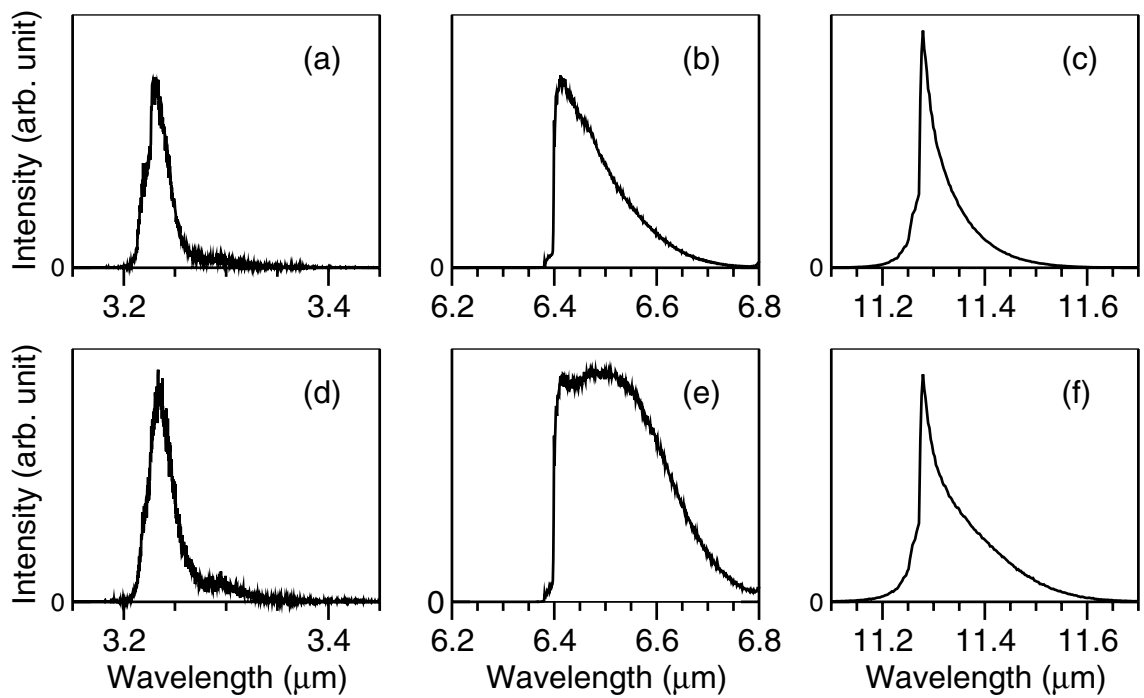

Fig. 2. Simulated emission spectra of the pyrene cation. In panels (a), (b), and (c), the blackbody temperature is $T_{\mathrm{bb}}=6000 \mathrm{~K}$; in panels $(\mathrm{d}),(\mathrm{e}),(\mathrm{f}), T_{\mathrm{bb}}=2 \times 10^{4} \mathrm{~K}$. The spectral ranges considered are 3.1-3.5 $\mu \mathrm{m}$ in panels (a) and (d), 6.2-6.8 $\mu \mathrm{m}$ in panels (b) and (e), and 11.1-11.7 $\mu \mathrm{m}$ in panels (c) and (f).

together with hydrogen dissociation, and one of them is randomly drawn with a probability proportional to its rate. The time and internal energy are updated, and the process is repeated until dissociation occurs or if time exceeds $10 \mathrm{~s} .10^{7}$ such $\mathrm{kMC}$ trajectories were performed in order to build reasonably smooth IR emission spectra.

In this article, only large band black-body excitations are considered. The absorption cross-section $\sigma(E)$ was adapted from experimental data (Salama \& Allamandola 1992; Biennier et al. 2004) in the low energy range $(E \leq 4 \mathrm{eV})$ and from theoretical works (Malloci et al. 2004) for $4<E \leq 12 \mathrm{eV}$. The initial electronic excitation $E^{*}$ under the blackbody radiation flux $\Phi(E)$ is randomly drawn at the beginning of each kMC trajectory from the probability $P\left(E^{*}\right) \propto \sigma\left(E^{*}\right) \Phi\left(E^{*}\right)$, after suitable normalization. As an illustration, the visible/UV absorption probability for a black-body temperature $T_{\mathrm{bb}}=6000 \mathrm{~K}$ is displayed in Figure 1a. At this temperature, absorption mainly occurs near $6 \mathrm{eV}$ due to large cross-sections of excited electronic states in this energy range. The dissociation efficiency is shown in Figure $1 \mathrm{~b}$ versus $T_{\mathrm{bb}}$. Dissociation is found to play a role in the photodynamics of the pyrene cation only when $T_{\mathrm{bb}}$ exceeds about $1000 \mathrm{~K}$. In the temperature range considered here, the dissociation efficiency is always lower than $40 \%$. As will be seen below, the dissociation involves hydrogen loss, which significantly alters the infrared emission spectral features. 


\section{Results and discussion}

For the sake of brevity, we focus on the evolution of IR emission spectral features as a function of the blackbody temperature associated to the stellar radiation flux. Three vibrational bands have been considered, namely " 3.3 " $\mu \mathrm{m}$ (C-H stretchings), "6.2" $\mu \mathrm{m}$ (C-C stretchings) and "11.2" $\mu \mathrm{m}$ (C-H out-of-plane bendings), owing to their astrophysical relevance. A more detailed analysis of the IR emission bands will be given in a forthcoming article. In Figure 2, the emission spectra for these three bands are shown for $T_{\mathrm{bb}}=6000 \mathrm{~K}$ and $2 \times 10^{4} \mathrm{~K}$.

The calculated emission spectra are broad, continuous, and strongly asymmetric, especially for the two softer bands where long wings on the red side are consistent with observations (Van Diedenhoven et al. 2004; Peeters et al. 2002). This spectral profile is in agreement with previous calculations in which spectral shifts and broadenings due to anharmonicity were extracted from experimental data (Pech et al. 2002). The asymmetry also increases with blackbody temperature, and the profile changes significantly in the case of the " 6.2 " $\mu \mathrm{m}$ band. All these effects originate from anharmonicities of the ground state potential energy surface.

On a more quantitative level, the spectral shift $\langle\lambda\rangle$ and the spectral width $\Delta \lambda$ have been computed as a function of $T_{\mathrm{bb}}$ using the first and second moments of the IR emission spectrum, respectively. The variations of $\langle\lambda\rangle$ and $\Delta \lambda$ with $T_{\mathrm{bb}}$ are represented in Figure 3 for the same three vibrational bands depicted in Figure 2. Comparing $\langle\lambda\rangle$ to the harmonic wavelength $\lambda_{k}^{(\mathrm{h})}$, the "11.2" band turns out to be less sensitive to anharmonicities than the two others bands. At very high blackbody temperatures, both the shift and width tend to constant values for the three modes. This is a consequence of hydrogen dissociation, which at high energies becomes an important channel very soon after excitation (see Fig. 1), thus quenching IR emission due to the concomitant strong evaporative cooling.

Finally, we note that the three simulated spectral widths obtained here for cationic pyrene are of the same order of magnitude as those observed in the majority of interstellar sources (Van Diedenhoven et al. 2004; Peeters et al. 2002). For the three bands considered so far (in decreasing wavelength), the observed widths are approximately equal to $0.20 \mu \mathrm{m}, 0.13 \mu \mathrm{m}$, and $0.04 \mu \mathrm{m}$, respectively, in agreement with Figure 3.

The observed spectral features seem rather unsensitive to the astrophysical objects in the class A (Peeters et al. 2002; Van Diedenhoven et al. 2004; Galliano et al. 2008). The observed IR band broadening is the consequence of the PAH size distribution. However, as demonstrated by the present calculations limited to cationic pyrene, the asymmetric spectral profiles are well reproduced when the PES anharmonicity is properly incorporated, especially for the " $11.2 " \mu \mathrm{m}$ band. The observed IR emission spectra could then be strongly influenced by the variety in the intrinsic spectral width of individual PAHs resulting from different anharmonicities. The present computational protocol offers a convenient way to address this issue for larger molecules on a quantitative footing. Work is currently in progress to analyse the corresponding size effects on IR emission spectroscopy. 

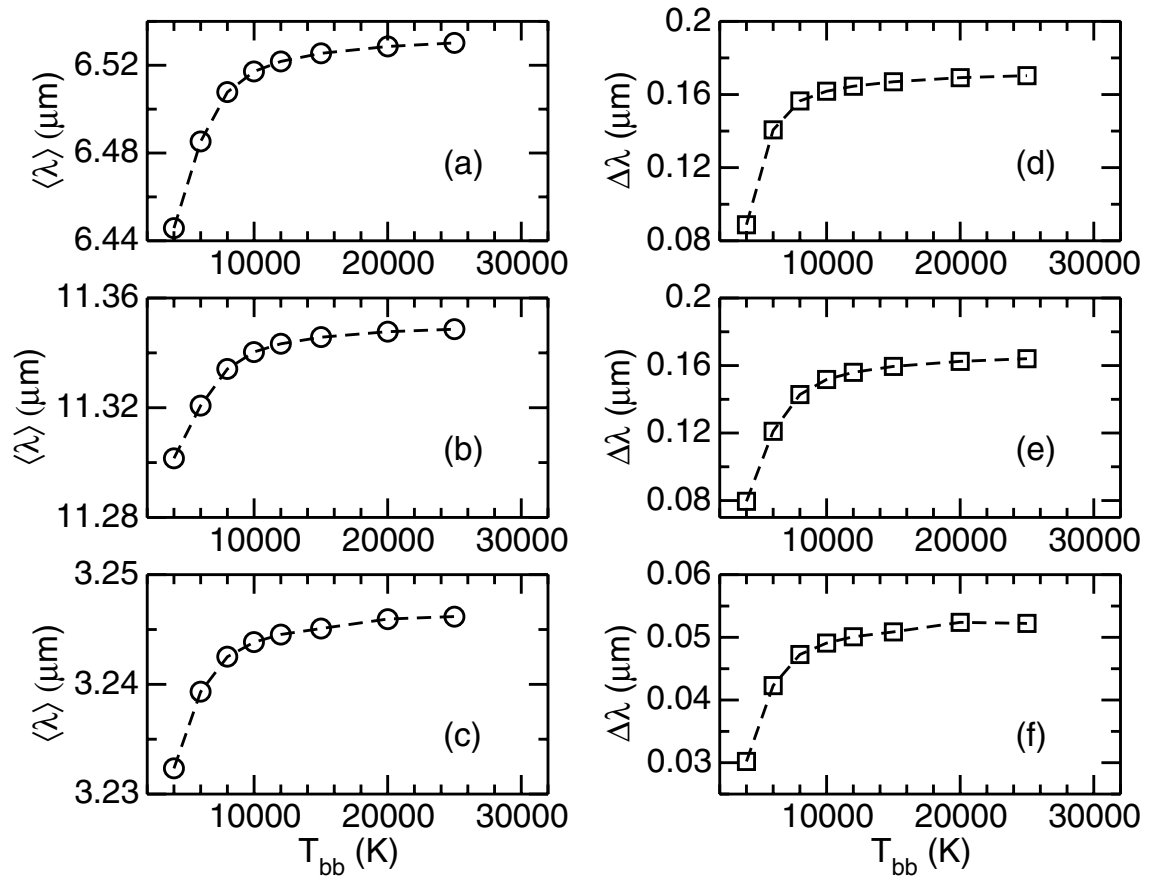

Fig. 3. Variations of the spectral shift $\langle\lambda\rangle$ (left panels) and width $\Delta \lambda$ (right panels) with increasing blackbody temperature, for the same three vibrational bands of the pyrene cation depicted in Figure 2. (a) and (d): $11.10 \mu \mathrm{m}$; (b) and (e): $6.25 \mu \mathrm{m}$; (c) and (f): $3.10 \mu \mathrm{m}$.

\section{References}

Allamandola, L.J., Tielens, A.G.G.M., \& Barker, J.R., 1989, ApJS, 71, 733

Barker, J.R., 1983, Chem. Phys., 77, 301

Basire, B., Parneix, P., \& Calvo, F., 2008, J. Chem. Phys., 129, 0811019

Basire, B., Parneix, P., Calvo, F., Pino, T., \& Bréchignac, Ph., 2009, J. Phys. Chem. A, 113, 6947

Basire, B., Parneix, P., \& Calvo, F., 2010, J. Phys. Chem. A, 114, 3139

Biennier, L., Salama, F., Gupta, M., \& O'Keefe, A., 2004, Chem. Phys. Lett., 387, 287

Brenner, J., \& Barker, J.R., 1992, ApJ, 388, L39

Cherchneff, I., \& Barker, J.R., 1989, ApJ, 341, L21

Cook, D.J., Schlemmer, S., Balucani, N., et al., 1996, Nature, 380, 227

Cook, D.J., \& Saykally, R.J., 1998, A\&A, 493, 793

Draine, B.T., \& Li, A.G., 2001, ApJ, 551, 807

Draine, B.T., \& Li, A.G., 2007, ApJ, 657, 810

Galliano, F., Madden, S.C., Tielens, A.G.G.M., Peeters, E., \& Jones, A.P., 2008, ApJ, 679,310 
Gillespie, D.T., 1978, J. Comput. Phys., 28, 395

Gillett, F.C., Forrest, W.J., \& Merrill, K.M., 1973, ApJ, 183, 87

Hony, S., Van Kerckoven, C., Peeters, E., et al., 2001, A\&A, 370, 1030

Joblin, C., Boissel, P., Léger, A., \& Défourneau, D., 1995, A\&A, 299, 835

Joblin, C., Toublanc, D., Boissel, P., \& Tielens, A.G.G.M., 2002, Mol. Phys., 100, 3595

Kim, H.S., Wagner, D.R., \& Saykally, R.J., 2001, Phys. Rev. Lett., 86, 5691

Kim, H.S., \& Saykally, R.J., 2002, ApJS, 143, 455

Léger, A., D'Hendecourt, L., \& Défourneau, D., 1989, A\&A, 216, 148

Malloci, G., Mulas, G., \& Joblin, C., 2004, A\&A, 426, 105

Mulas, G., 1998, A\&A, 338, 243

Pech, C., Joblin, C., \& Boissel, P., 2002, A\&A, 388, 639

Pino, T., Parneix, P., Calvo, F., \& Bréchignac, Ph., 2007, J. Phys. Chem. A, 111, 4450

Peeters, E., Hony, S., Van Kerckoven, C., et al., 2002, A\&A, 390, 1089

Puget, J.L., \& Léger, 1989, ARA\&A, 27, 161

Salama, F., \& Allamandola, L.J., 1992, Nature, 358, 42

Schutte, W.A., Tielens, A.G.G.M., \& Allamandola, L.J., 1993, ApJ, 415, 397

Van Diedenhoven, B., Peeters, E., Van Kerckoven, C., et al., 2004, ApJ, 611, 928

Wang, F., \& Landau, D.P., 2001, Phys. Rev. Lett., 86, 2050

Williams, R.M., \& Leone, S.R., 1995, ApJ, 443, 675 